\title{
Inhibition of mTOR or MAPK ameliorates vmhcl/myh7 cardiomyopathy in zebrafish
}

\author{
Haisong Bu, ${ }^{1,2}$ Yonghe Ding, ${ }^{1}$ liarong Li, ${ }^{1,3}$ Ping Zhu, ${ }^{1}$ Yu-Huan Shih, ${ }^{1}$ Mingmin Wang, ${ }^{1,4}$ Yuji Zhang, ${ }^{5}$ \\ Xueying Lin, ${ }^{1}$ and Xiaolei $\mathrm{Xu}^{1}$ \\ 'Department of Biochemistry and Molecular Biology, Department of Cardiovascular Medicine, Mayo Clinic, Rochester, \\ Minnesota, USA. 'Department of Cardiothoracic Surgery, Xiangya Hospital, Central South University, Changsha, China. \\ ${ }^{3}$ Department of Cardiovascular Surgery, The Second Xiangya Hospital of Central South University, Changsha, China. \\ ${ }^{4}$ Dongzhimen Hospital, Beijing University of Chinese Medicine, Beijing, China. ${ }^{5}$ Department of Epidemiology and Public \\ Health, University of Maryland School of Medicine, Baltimore, Maryland, USA.
}

\begin{abstract}
Myosin heavy chain 7 (MYH7) is a major causative gene for hypertrophic cardiomyopathy, but the affected signaling pathways and therapeutics remain elusive. In this research, we identified ventricle myosin heavy chain like (vmhcl) as a zebrafish homolog of human $\mathrm{MYH7}$, and we generated vmhcl frameshift mutants. We noted vmhcl-based embryonic cardiac dysfunction (VEC) in the vmhcl homozygous mutants and vmhcl-based adult cardiomyopathy (VAC) phenotypes in the vmhcl heterozygous mutants. Using the VEC model, we assessed 7 known cardiomyopathy signaling pathways pharmacologically and 11 candidate genes genetically via CRISPR/Cas 9 genome editing technology based on microhomology-mediated end joining (MMEJ). Both studies converged on therapeutic benefits of mTOR or mitogen-activated protein kinase (MAPK) inhibition of VEC. While mTOR inhibition rescued the enlarged nuclear size of cardiomyocytes, MAPK inhibition restored the prolonged cell shape in the VEC model. The therapeutic effects of mTOR and MAPK inhibition were later validated in the VAC model. Together, vmhcl/myh7 loss of function is sufficient to induce cardiomyopathy in zebrafish. The VEC and VAC models in zebrafish are amenable to both efficient genetic and chemical genetic tools, offering a rapid in vivo platform for discovering candidate signaling pathways of $M Y H 7$ cardiomyopathy.
\end{abstract}

Authorship note: $\mathrm{HB}$ and $\mathrm{YD}$ contributed equally to this work.

Conflict of interest: The authors have declared that no conflict of interest exists.

Copyright: () 2021, Bu et al. This is an open access article published under the terms of the Creative Commons Attribution 4.0 International License.

Submitted: August 17, 2021 Accepted: November 3, 2021 Published: December 22, 2021

Reference information: JCI Insight. 2021;6(24):e154215.

https://doi.org/10.1172/jci. insight.154215.

\section{Introduction}

Cardiomyopathy $(\mathrm{CM})$ refers to a group of heterogeneous cardiac muscle diseases that are categorized into hypertrophic CM (HCM), dilated CM (DCM), and restrictive CM (RCM) (1-4). Genetic contributions to CM have been identified, and more than 100 genes have been linked to different types of CM (5, 6). Animal models have been generated and used for discovering key signaling pathways and therapeutic strategies. At least $7 \mathrm{CM}$ signaling pathways with therapeutic potential have been identified, including mitogen-activated protein kinase (MAPK) signaling, mTOR signaling, $\beta$-adrenergic receptor signaling, phosphodiesterase 5 (PDE5) signaling, histone deacetylase (HDAC) signaling, $\mathrm{Ca}^{2+} /$ calmodulin-dependent kinase II signaling, and calcineurin-nuclear factor of activated T cells (Cn-NFAT) signaling pathways (7-9). For example, mTOR is a serine/threonine protein kinase that plays a pivotal role in regulating proteostasis in cardiomyocytes (10-12); partial mTOR inhibition through either pharmacological or genetic approaches exerts cardioprotective effects on several types of cardiomyopathies, including lamp2-associated HCM (13), bag3-associated and lamin A/C-associated DCM (14, 15), and anemia- and doxorubicin-induced cardiomyopathies (DICs) (16). In contrast, MAPKs have been found to activate in response to almost every stress- and agonist-induced hypertrophic stimulus, and uniquely regulate the balance between eccentric and concentric growth of the heart $(17,18)$. Whereas activation of MAPK results in eccentric hypertrophy and promotes myocyte lengthening, inhibition of the extracellular signal-regulated kinase (ERK) pathway results in an attenuated hypertrophic response to pressure overload (19).

$M Y H 7$, also termed $\beta$-myosin heavy chain, was the first identified CM-causative gene, which later was determined to be responsible for $\sim 18 \%$ of HCM cases (20-22). In humans, MYH7 is located in tandem with MYH6 on chromosome 14, with $M Y H 7$ being the predominant adult isoform that is located upstream of $M Y H 6$. In mice, $M y h 7$ and Myh6 are also located in tandem on chromosome 14; however, the upstream $M y h 7$ gene 
is mainly expressed in the embryonic heart, whereas Myh6 is expressed in the adult heart (23), an opposite pattern to that in humans. Mechanistic studies of MYH7 R403Q, which causes a common and particularly malignant form of inherited HCM (24), yield conflicting information from studies in mice versus those in large mammals/humans. Earlier results with human cardiac myosin showed a loss of function $(25,26)$, which is supported by recent transgenic studies in rabbits carrying an R403Q mutation in $M Y H(22,27,28)$. In contrast, transgenic studies in mice suggested a gain-of-function nature of $M y h 7 \mathrm{R} 403 \mathrm{Q}(25,29)$. Whether any known CM pathways are effective at ameliorating $M Y H 7 \mathrm{CM}$ remains untested, to our knowledge.

Zebrafish (Danio rerio) is an emerging vertebrate model for $\mathrm{CMs}$, and titin and troponin $\mathrm{T}$ mutants were considered as the first embryonic CM models $(30,31)$. However, it remains unclear whether these embryonic models faithfully recapitulate pathogenesis in human CMs, a group of progressive diseases with late onset. To address this concern, adult zebrafish CM models have been developed, such as those induced by anemia, doxorubicin, and diabetes (32). The advent of genome editing technology enabled the generation of inherited CM models with mutations in known causative genes, such as titin $(33,34)$, bag3 (14), and lamp2 (13). Compared to rodents, zebrafish are amenable to more efficient genetics and chemical genetic tools. A CRISPR/ Cas9 genome editing technology based on microhomology-mediated end joining (MMEJ) was recently established, and this approach has enabled the generation of predictable biallelic loss-of-function mutants in F0 fish to allow a rapid establishment of genotype-phenotype relationships (35). Owing to their high throughput and excellent permeability, zebrafish embryos have also been successfully utilized to conduct pharmacological screens and identify compounds with therapeutic capacity for doxorubicin-induced cardiotoxicity and the induction of anf, a molecular marker of $\mathrm{CM}(36,37)$. Moreover, a mutagenesis screen-based strategy has been established to discover novel genetic factors and therapeutic target genes for CMs $(3,38,39)$.

Encouraged by these powerful genetic and chemical genetic capabilities, we explored zebrafish as an alternative animal model for MYH7 CM. Utilizing transcription activator-like effector nuclease (TALEN) technology, we generated frameshift mutants for the ventricle myosin heavy chain-like ( $v m h c l$ gene, also known as $m y h 7 l$. We provided evidence of its identity as an $M Y H 7$ homolog and established both $v m h c l$ homozygous embryos and heterozygous adults as models for MYH7 CM. By performing both compound testing and genetic assessments using the MMEJ-based CRISPR/Cas9 genome editing technology in the embryonic model, we identified the therapeutic effects of mTOR and MAPK inhibition. Our data established zebrafish as an in vivo model for mechanistic study and therapeutic development for MYH7 CM.

\section{Results}

Depletion of vmhcl, a candidate MYH7 homolog, induces cardiac dysfunction in zebrafish embryos. Our previous transcriptome studies identified 9 myh homologs in the zebrafish genome (40), among which atrial myosin heavy chain $(a m h c)$, ventricular myosin heavy chain $(v m h c)$, and ventricular myosin heavy chain-like $(v m h c l)$ are 3 predominant cardiac homologs, accounting for more than $99 \%$ of transcripts in the zebrafish heart. The $v m h c l$ gene encodes a protein that shares $85.9 \%$ similarity with the human $M Y H 7$ protein (Supplemental Figure 1; supplemental material available online with this article; https://doi.org/10.1172/jci. insight.154215DS1). Similar to $v m h c$ but not $a m h c, v m h c l$ is specifically expressed in the ventricle, as indicated by in situ whole-mount staining (Supplemental Figure 2, A and B). Consistent with our transcriptome data indicating that $v m h c$ is a predominant embryonic $m y h$ isoform and $v m h c l$ is a predominant adult isoform (40), we found that the expression of the $v m h c$ transcripts gradually decreased after 10 weeks of age and were undetectable after 13 weeks; in contrast, cardiac expression of $v m h c l$ transcripts remained strong until at least 13 weeks (Supplemental Figure 2C). In zebrafish, $v m h c l$ and $v m h c$ are located in tandem on chromosome 2, with $v m h c l$ located approximately $4.4 \mathrm{~kb}$ upstream of $v m h c$ (Supplemental Figure 2D) (40). Because of the similarity with MYH7/MYH6 in humans, where the upstream gene is the predominant adult cardiac isoform and the downstream gene is mainly expressed in embryonic hearts (40), we propose that vmhcl is an MYH7 homolog in zebrafish and vmhc is an MYH6 homolog.

We then generated $v m h c^{e 13}$, a $v m h c l$ mutant harboring an 8-nucleotide deletion (Figure 1A and Supplemental Figure 3) by targeting the 13th exon of $v m h c l$ using TALEN technology. The expression of the $v m h c l$ transcript was reduced by $39 \%$ in $v m h c^{1213 /+}$ and $90 \%$ in $v m h c^{121 / e 13}$ zebrafish (Figure 1B), likely due to nonsense-mediated RNA decay. The $v m h l^{1213 / e 13}$ homozygous mutants manifested pericardial edema starting at 3 days after fertilization (dpf) (Figure 1C), concurrent with significantly enlarged chamber size (Figure 1, $\mathrm{D}$ and $\mathrm{E}$ ). The cardiac pump function, as measured by fractional shortening (FS), was significantly reduced in the $v m h c l^{e 13 / e 13}$ homozygous hearts as well (Figure $1 \mathrm{~F}$ ). Because $v m h c l$ encodes a sarcomeric protein, we 
assessed the sarcomere structure in $v m h c^{113 / e 13}$ zebrafish. Striated thick and thin filaments were disrupted in the homozygous mutants, as revealed by immunostaining using an anti-myosin heavy chain 1 A (MYH1A) (F59) or anti-troponin $\mathrm{T}$ antibody, respectively (Figure 1, G and $\mathrm{H}$ ). These sarcomeric phenotypes were confirmed by transmission electronic microscopy (TEM) analysis (Figure 1I). Consistent with the ventricle-specific expression pattern of $\mathrm{vmhcl}$, these sarcomeric abnormalities occurred only in the ventricle but not in the atrium (Supplemental Figure 4, A, B, and D). The enlarged atrium size (Figure 1E), manifesting as reduced cardiomyocyte cell density and increased cardiomyocyte cell size (Supplemental Figure 4), was considered to be a secondary effect of ventricule dysfunction. Taken together, these results suggest that depletion of $v m h c l$ yields a $v m h c l$-based embryonic cardiac dysfunction (VEC) model.

vmhcl haploinsufficiency results in CM-like phenotypes in adult zebrafish. Next, we studied the vmhclel3/+ fish. At the protein level, the levels of $M y h$ bands of approximately $200 \mathrm{kDa}$ in size were reduced in the $v m h$ $\mathrm{cl}^{\mathrm{el} 3 /+}$ mutant at 6 months (Supplemental Figure 5, A and B). At the mRNA level, vmhcl haploinsufficiency manifested as reduced $v m h c l$ transcripts that were compensated by increased expression of the $v m h c$ and $a m h c$ transcripts, without affecting the expression of myh7ba and myh7bb,2 minor zebrafish cardiac $m y h$ homologs (Figure 2A and Supplemental Figure 5C). We noted significant decreases in the ejection fraction (EF) and FS, as measured by high frequency echocardiography, in the $v m h c^{13 /++}$ mutant zebrafish at 8 months (Figure 2, B and C). The reduction in cardiac pump function was largely ascribed to the increased end-systolic volume/body weight (ESV/BW) ratio, while the end-diastole volume/BW (EDV/BW) ratio remained unchanged. Consistent with this observation, we noted an enlarged heart chamber size, as indicated by the significantly increased ventricular surface area/BW (VSA/BW) index in the $v m h{ }^{1013 /+}$ mutant

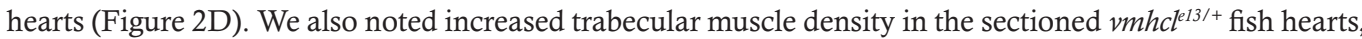
as determined by H\&E staining analysis (Figure 2E). At the molecular level, the expression of cardiac remodeling markers, such as natriuretic peptide $\mathrm{B}(n p p b)$ and natriuretic peptide A (nppa), was significantly elevated in the $v m h c l^{13 /+}$ mutant fish (Figure 2A). Exercise capacity, an important clinical index of heart failure in human patients, was also compromised in the $v m h c^{13 /+}$ mutant fish (Figure $2 \mathrm{~F}$ ). The $v m h c c^{13 /+}$ mutant fish started to die at 8 months old, and only approximately $60 \%$ of fish survived to 1.5 years of age (Figure $2 \mathrm{G}$ ). In summary, the adult $v m h \mathrm{cl}^{\mathrm{el3} /+}$ zebrafish manifested CM-like phenotypes and were designated as a vmhcl-based adult CM (VAC) model.

Compound and genetic screens in the VEC model identified mtor and mapk3 as candidate modifier genes. To explore the use of the VEC model for identifying signaling pathways with therapeutic potential, we assessed small-molecule inhibitors of 7 known CM signaling pathways (Table 1) (7). Effective doses of these small-molecule inhibitors were determined to avoid toxic effects $(38,41-44)$. At 5 dpf, 3 of 7 inhibitors (PD0325901, rapamycin, and carvedilol) mitigated the phenotypes in $v m \mathrm{mhc}^{\text {el3/el3 }}$ zebrafish, as indicated by significantly reduced percentages of homozygous $v m h c^{13 / e 13}$ fish with severe pericardial edema (Figure 3 , A and B, and Supplemental Figures 6 and 7), partially restored cardiac function (Figure 3C), and survival (Supplemental Figure 6B). Although none of these inhibitor treatments allowed homozygous $v_{m h c l^{e 13 / e 13}}$ zebrafish to survive to adulthood (Supplemental Figure 6B), the transient modulation of cardiac edema severity and cardiac function suggests that CM pathways can be studied in the VEC model.

Encouraged by our success with a compound-based pathway assessment (38), we then analyzed these signaling pathways genetically using the MMEJ-based CRISPR/Cas9 genome editing technology that enables rapid establishment of geneotype-phenotype relationships in F0 fish $(45,46)$. We selected 11 target genes from 7 known CM signaling pathways that could exert therapeutic effects on certain types of CM (Table 2) (7, 8), designed MMEJ-based sgRNAs for these genes, injected different doses of sgRNAs into zebrafish embryos at the 1-cell stage, and optimized doses by measuring resultant KO scores (Table 2 and Supplemental Table 1). We then injected sgRNAs into the offspring of $v m h c^{1213 /+}$ incrosses. We found that injection of sgRNAs for the mtor or mapk3 genes, but not the other 9 target genes, mitigated cardiac phenotypes in the $v_{m h} c^{e 13 / e 13}$ homozygous mutant, as indicated by rescuing effects on cardiac edema (Figure 4A), survival (Figure 4C), ventricular chamber volume (VCV) (Figure 4D), and percent FS as well (Figure 4E). Notably, the rescuing effects of mtor inhibition on FS were marginal; injection of sgRNAs for the mapk3, but not the mtor, also partially restored the disrupted sarcomere structure in the $v m h c^{121 / e 13}$ homozygous mutant (Figure 4B).

To confirm these observations from transient genetic studies, we then generated the $v m h c^{1213 / e 13} m t o r^{x u 015 /+}$

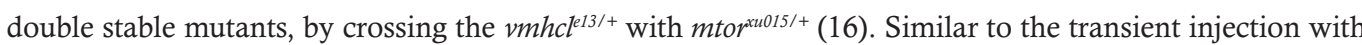
the mtor MMEJ sgRNA, mtor haploinsufficiency rescued the phenotypes of $v m h c^{13 / e 13} \mathrm{fish}$, as indicated by 
A

vmhcl

WT

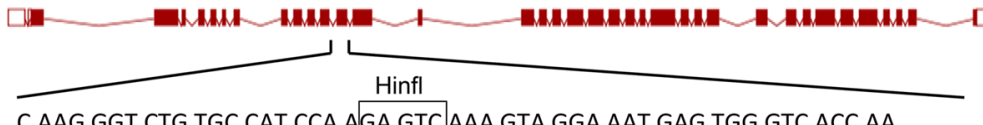

vmhc/e13

C AAG GGT CTG TGC CAT CCA AGA GTCAAA GTA GGA AAT GAG TGG GTC ACC AA

$\begin{array}{llllllllllllllllllllllll}K & G & L & C & H & P & R & V & K & V & G & N & E & W & V & T\end{array}$

$\begin{array}{lllllllllllll}K & G & L & C & H & P & & S & R & K & * & (P 403 f s 3 *)\end{array}$

B
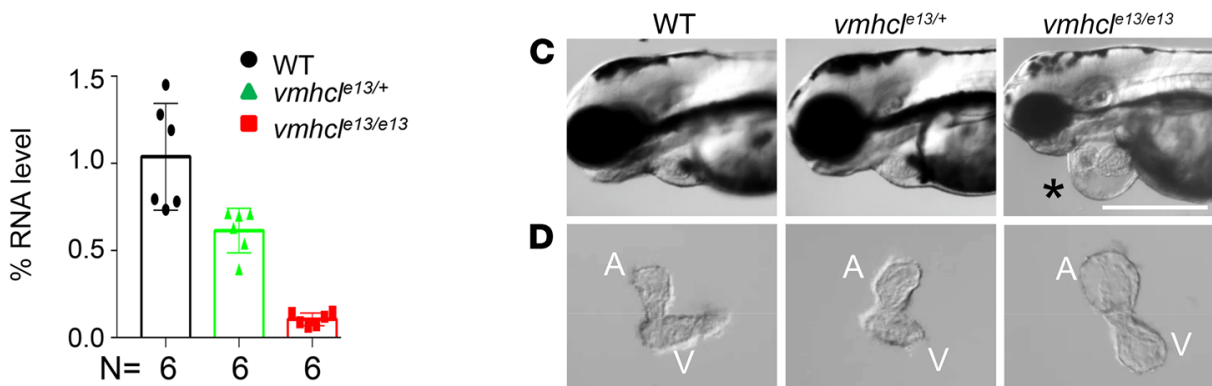

D
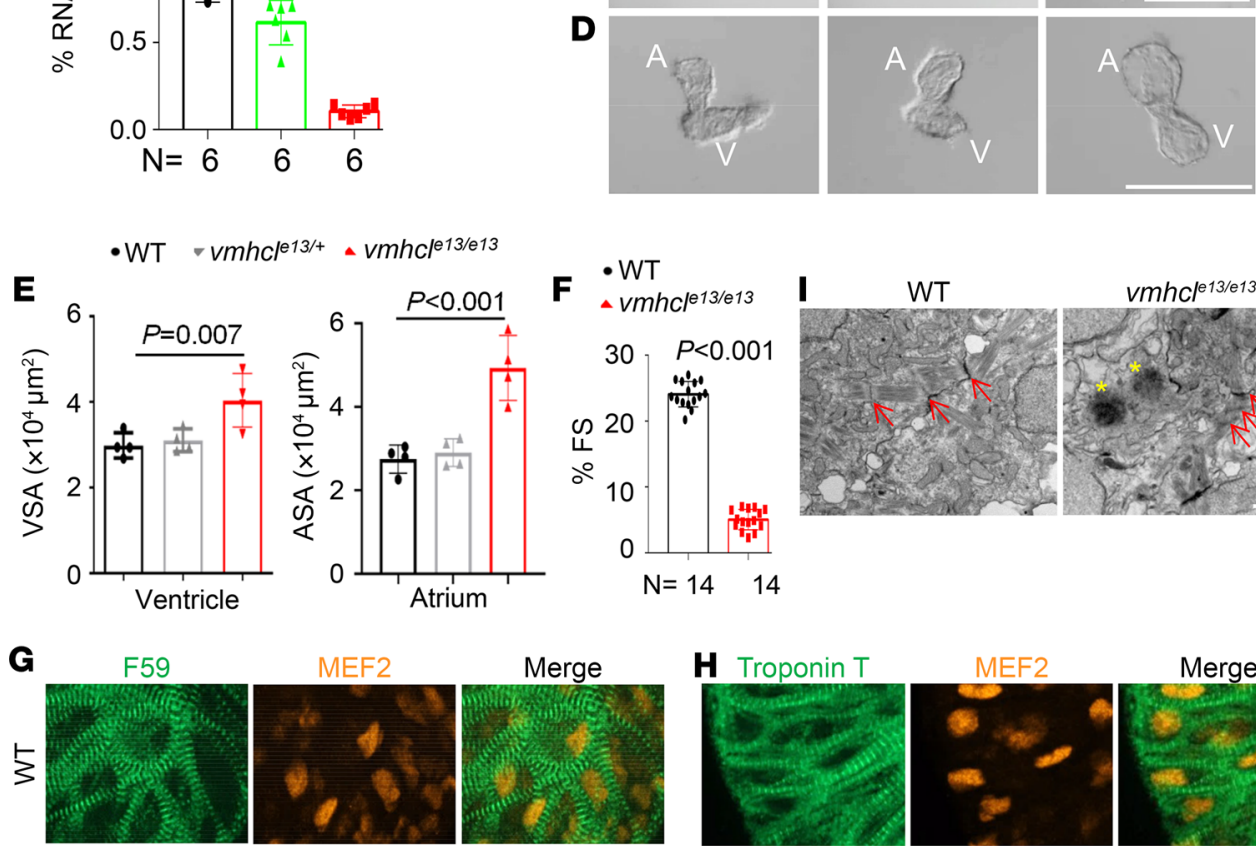

WT
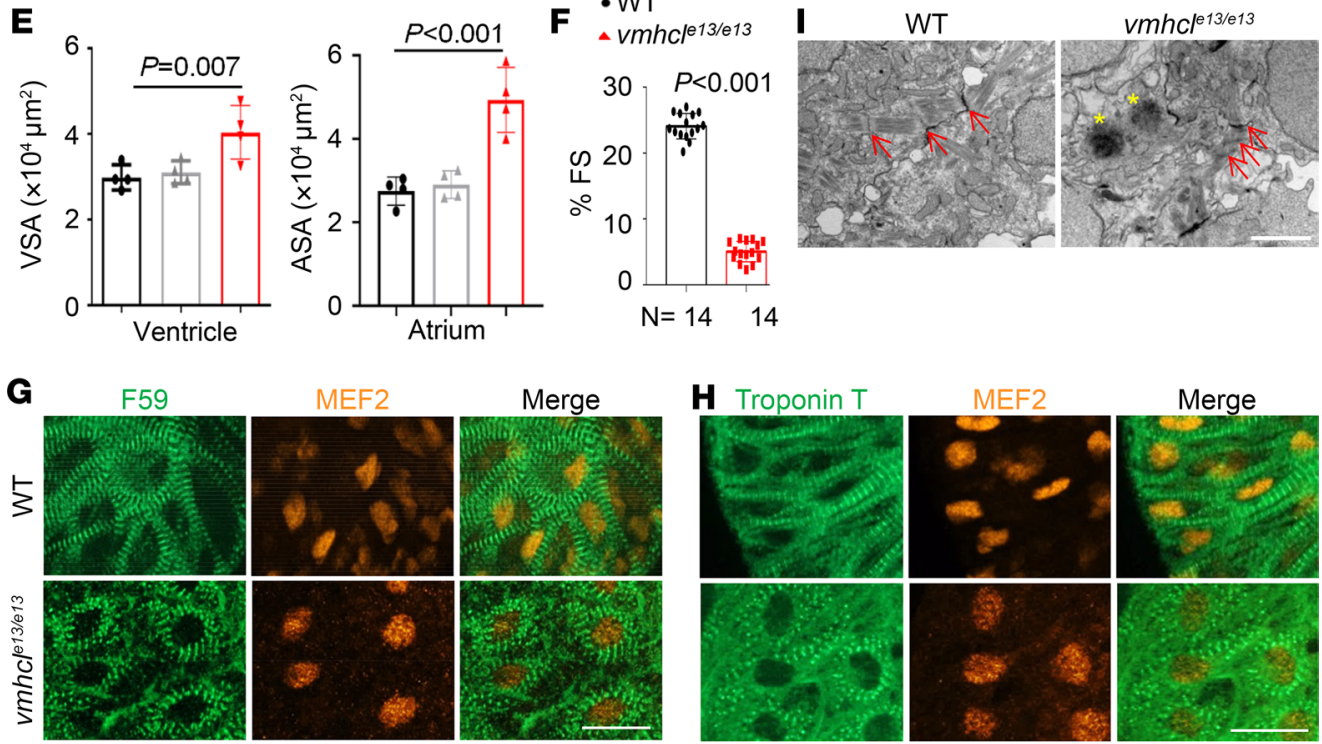

Figure 1. Depletion of vmhcl disrupts sarcomere integrity and results in a VEC model. (A) Schematics of the vmhcl mutant allele generated using TALEN. The Hinfl restriction enzyme recognition site used for genotyping is boxed. Dashed lines indicate deleted nucleotides. The asterisk indicates an early translational stop codon. $\mathrm{fs}$, frameshift. (B) Quantitative reverse transcription PCR (RT-PCR) revealed reduced expression of the transcript in both heterozygous (vmhcle13/+) and homozygous (vmhc/e13/e13) mutants. (C-E) Representative images and quantification of the VSA and atrial surface area (ASA) in fish at $3 \mathrm{dpf}$. The asterisk indicates edema. A, atrium; V, ventricle; $n=4$; 1 way ANOVA. (F) Percent FS of the vmhc/ ${ }^{13 / 213}$ mutant compared to WT control at $3 \mathrm{dpf} . n=14$; data are presented as the mean \pm SD. Paired 2-tailed Student's $t$ tests were used for statistical analyses. ( $\mathbf{G}$ and $\mathbf{H}$ ) The vmhc ${ }^{e^{13 / 213}}$ mutants exhibited disrupted sarcomere structure at $3 \mathrm{dpf}$. Fluorescence immunostaining using anti-myosin heavy chain 1 (F59) and anti-troponin T antibodies are shown. (I) Transmission electron microscope (TEM) images confirmed the disrupted sarcomere structure in the $v m h c^{113 / 13}$ mutants. Arrows indicate sarcomeric Z-discs in the vmhc/ ${ }^{13 / 213}$ mutant compared to WT control. Asterisks point to degenerated sarcomere in the vmhcle13/e13 mutant. Scale bars: $500 \mu \mathrm{m}$ in C $200 \mu \mathrm{m}$ in $\mathbf{D}, 10 \mu \mathrm{m}$ in $\mathbf{G}$ and $\mathbf{H}$, and $2 \mu \mathrm{m}$ in $\mathbf{I}$.

the rescued VCV and increased survival (Supplemental Figure 8, A, B, and D). Similar to genetic studies in F0, the rescuing effects on cardiac function were not significant (Supplemental Figure 8C). To generate stable mutants for mapk3, we raised F0 fish injected with a mapk3 targeting sgRNA to adults, selected fish predominantly harboring the 4 bp nucleotide deletion in their genome (Supplemental Figure 9, A and $\mathrm{B}$ ), and incrossed them to obtain F1 offspring. We identified F1 fish harboring a stable mapk3 mutation and designated these fish as mapk $3^{2-F 1}$. Consistent with our genetic analysis in F0, we detected therapeutic 
A

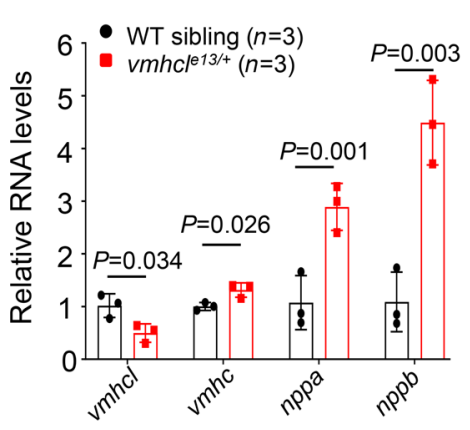

B
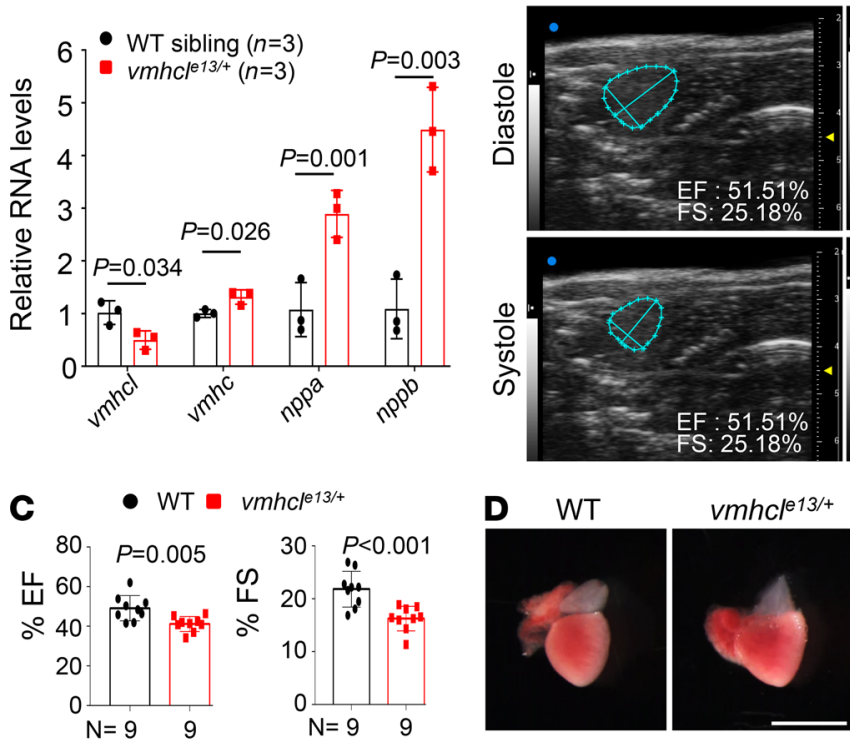

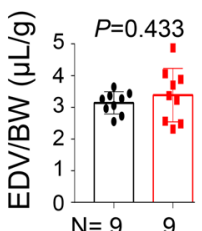

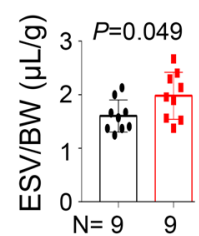

\section{$\mathbf{F}$}

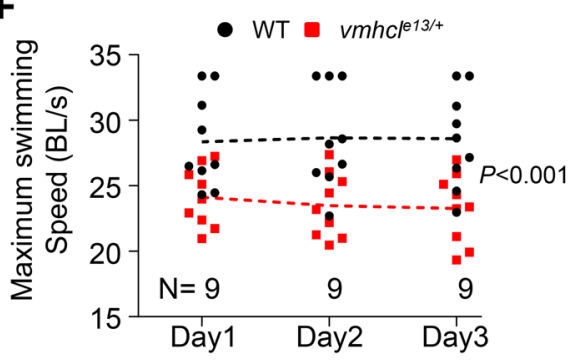

D WT

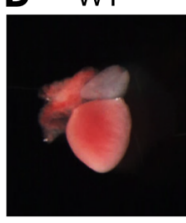

vmhc/e13/+
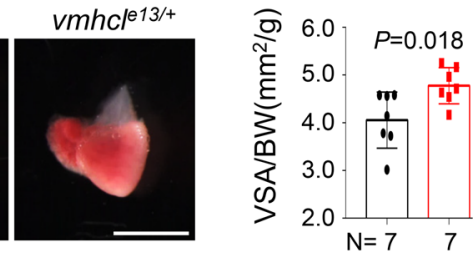

E WT
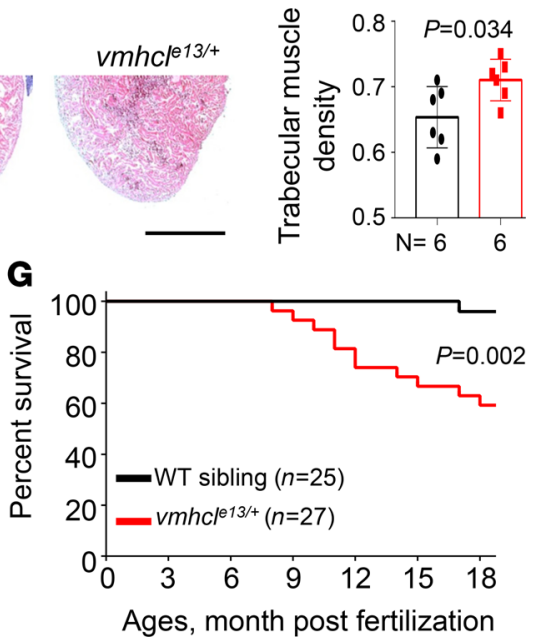

Figure 2. Haploinsufficiency of vmhcl results in CM in adult zebrafish. (A) Quantitative RT-PCR analysis of CM molecular markers. $n=3$ biological replicates; 2 tailed Student's $t$ test. (B) Representative echocardiography images of WT controls and vmhcle13/+ mutants at diastole and systole. (C) Quantification of cardiac function indices measured using echocardiography in the vmhc|e13/+ mutant and WT control at 8 months. $n=9$; data are presented as the mean \pm SD; unpaired 2 tailed Student's $t$ test. (D) Representative images of isolated hearts and quantification of the VSA normalized to the BW of fish at 8 months. $n=7$; unpaired 2 tailed Student's $t$ test. (E) Representative images of H\&E staining in the apex area and quantification of trabecular muscle density in fish at 8 months. $n=$ 6; unpaired 2 tailed Student's $t$ test. (F) Maximum swimming speed of fish at 8 months. $n=9$; 2-way ANOVA. (G) Kaplan-Meier survival curves of vmhcl mutant fish and WT controls. $n=25-27$; log-rank test; data are presented as the mean \pm SD. Scale bars: $2 \mathrm{~mm}$ in $\mathbf{D}$ and $300 \mu \mathrm{m}$ in $\mathbf{E}$.

effects of mapk $3^{2-F 1}$ on the $v m h c^{1213 / e l 3}$ phenotype, as evidenced by significantly reduced VCV and improved

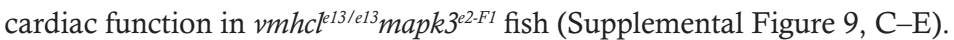

Distinct cellular effects of mtor inhibition and mapk3 inhibition on the VEC model. To discern the functions of mtor inhibition and mapk 3 inhibition, we examined cellular changes in the VEC model. Using an anti- $\beta$-catenin antibody to define the cell border and an anti-myocyte enhancer factor-2 (anti-Mef2) antibody to label the cardiomyocyte nuclei (Figure 5A), we noted a marked change in cardiomyocyte morphometry in the outer curvature (OCR) of the ventricles in the VEC model at $3 \mathrm{dpf}$. Compared to a prolonged shape in wild-type cardiomyocytes, these mutant cardiomyocytes were wider but shorter, manifesting a rounded shape (Figure 5 , B-D). The cardiomyocyte area was significantly enlarged, and the cardiomyocyte density was lower, suggesting cardiomyocyte hypertrophy (Figure 5, E-G). We also noticed an increase in the size of cardiomyocyte nuclei. 
Table 1. A summary of small-molecule inhibitors of 7 known CM signaling pathways

\begin{tabular}{|c|c|c|c|}
\hline Pathway signaling & Drug & Concentration & Phenotype (rescued) \\
\hline $\mathrm{Ca}^{2+} /$ calmodulin-dependent kinase II signaling & Rimacalib (SMP-114) & $10 \mu \mathrm{M}$ & $E, C$ \\
\hline Cn-NFAT signaling & Cyclosporine A & $1 \mu \mathrm{M}$ & $E, C$ \\
\hline MAPK signaling & PD0325901 & $100 \mathrm{nM}$ & $E, C$ \\
\hline HDAC signaling & Vorinostat & $20 \mu \mathrm{M}$ & $E, C$ \\
\hline mTOR-autophagy signaling & Rapamycin & $100 \mathrm{nM}$ & $E, C$ \\
\hline
\end{tabular}

Edema, E; Cardiac dysfunction, C.

Injection of an MMEJ sgRNA against mtor effectively rescued the size of cardiomyocyte nuclei but did not affect cardiomyocyte morphology, size, or density (Figure 5, B-G). In contrast, injection of an MMEJ sgRNA against mapk3 specifically restored the prolonged cardiomyocyte shape by increasing the length and decreasing the width. The cardiomyocyte area, density, and nuclei size were not affected (Figure 5, B-G). Together, these data suggested distinct mechanisms underlying the therapeutic effects of mtor and mapk3 inhibition on the VEC model (Figure $5 \mathrm{H})$.

Therapeutic effects of mTOR or MAPK inhibition are conserved in the VAC model. Lastly, we tested whether $\mathrm{CM}$ signaling pathways identified in the VEC model were conserved in the VAC model. At the molecular level, we detected activated mTOR signaling, as indicated by hyperphosphorylation of mTOR, activation of pS6, and reduced levels of the LC3-II protein in the $v m h c^{\text {lel3/+ }}$ mutants (Figure 6, A and B). In the $v m h$ $\mathrm{cl}^{\mathrm{el3/+}}$ mtor ${ }^{\mathrm{ru} 015 /+}$ double mutants, mtor ${ }^{\mathrm{ru} 015+/-}$ effectively reverted these molecular changes (Figure 6, A and B). Consequently, mtor inhibition significantly rescued the reduced cardiac function and enlarged heart size; nonetheless, the increased trabecular muscle density was not rescued (Figure 6, C-H). Similarly, we also noted partially restored cardiac function in the adult $v m h l^{e l 3 /+} m a p k 3^{M J-F 0}$ double mutants (Supplemental
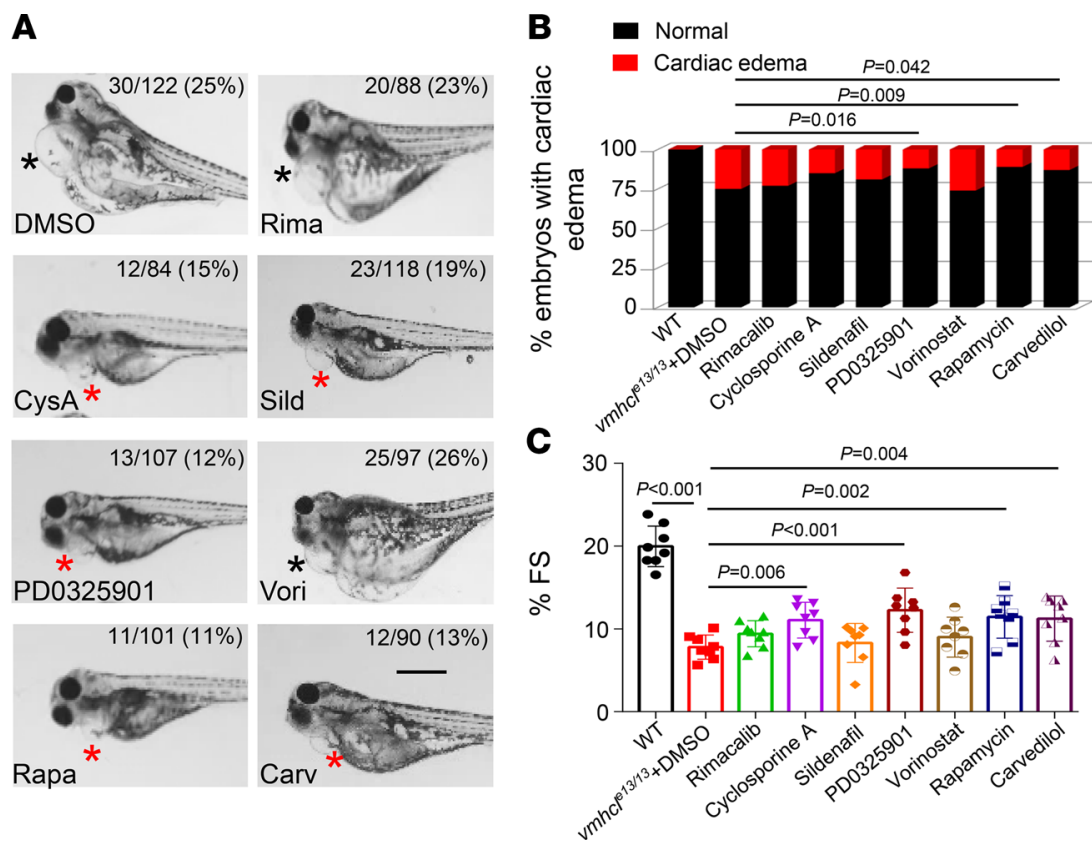

Figure 3. A screen of compounds targeting 7 known CM pathways identified therapeutic compounds for VEC. (A and B) Representative images and percentage of $v m h c^{\mid 13 / 213}$ mutant fish with the indicated phenotypes after administration of the compounds or DMSO control at 5 dpf. Black stars indicate severe edema, and red stars indicate mild edema. It is anticipated that $25 \%$ of vmhc/e13/+ incross offspring are homozygous vmhc/e13/213, which manifest severe pericardial edema (black stars). Administration of 5 different drugs reduced the percentage of $v m h c^{133 / 213}$ mutants with edema to less than $20 \%$; 3 of these drugs reduced the percentage with edema with statistical significance $(P<0.05) . n=84-122 ; \chi^{2}$ test; scale bar: $500 \mu \mathrm{m}$. (C) Percent FS of the $\left.v m h c\right|^{13 / 1 / 13}$ mutants after administration of the compounds compared to WT control at $5 \mathrm{dpf} . n=8$; data are presented as the mean \pm SD; 1 -way ANOVA. 
Table 2. A summary of 11 genes from 7 known CM signaling pathways

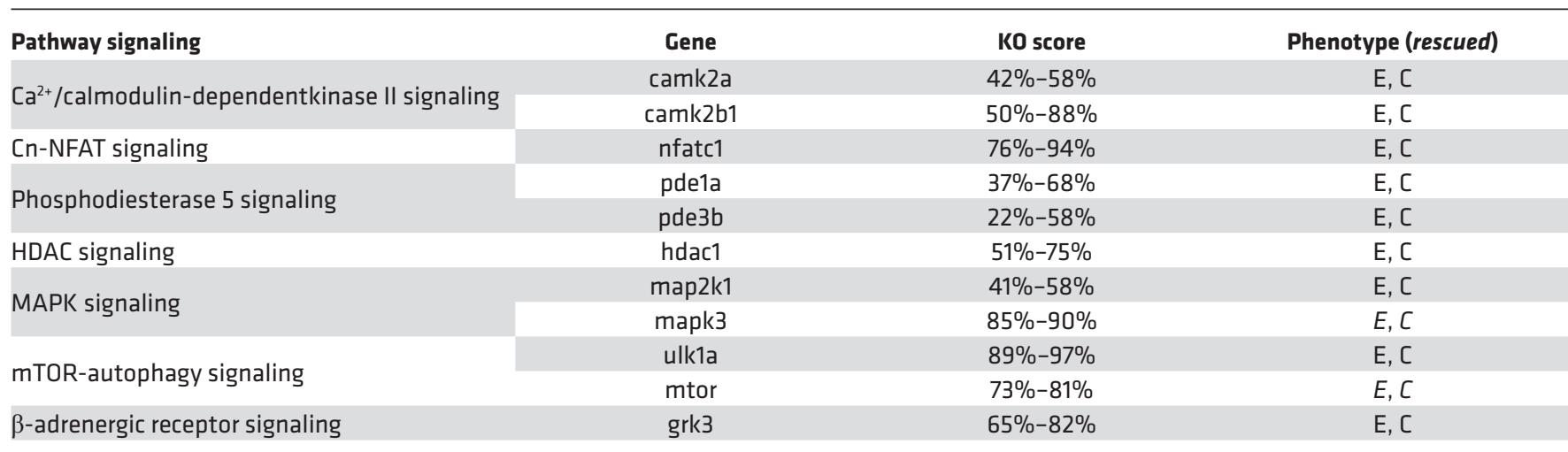

Edema, E; Cardiac dysfunction, C.

Figure 10, A and B). Interestingly, we were able to identify $2 v m h l^{13 / e 13} m a p k 3^{M J-F 1}$ homozygous fish that survived to adulthood after genotyping approximately 200 adult fish generated from the vmhcle13/+ and map-

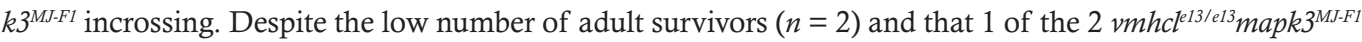
homozygous fish displayed an enlarged ventricle and atrium (Supplemental Figure 10, C-E), this observation strongly suggests a rescuing effect of MAPK inhibition on the embryonic lethality of $v m$ ch $^{\text {el3/el3 }}$ homozygosity. Taken together, these data confirmed the therapeutic effects of mTOR or MAPK inhibition on the zebrafish VAC model.
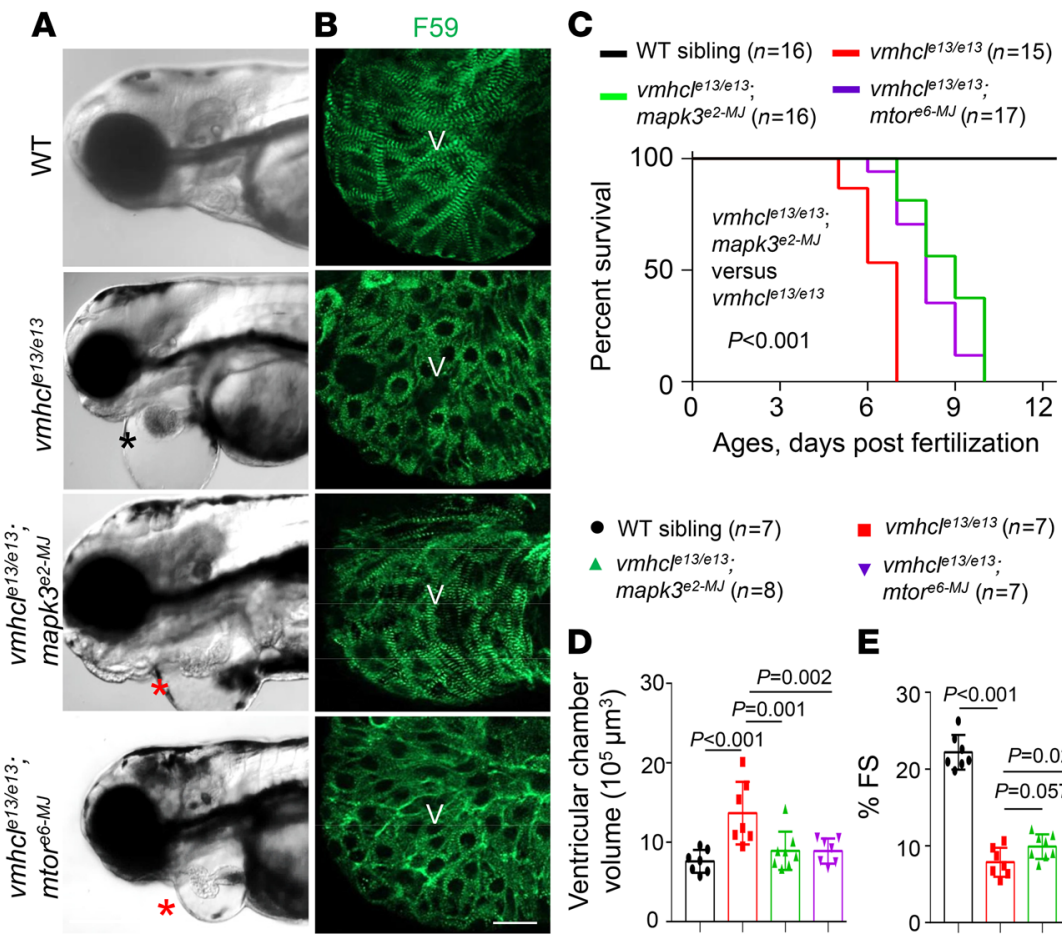

Ages, days post fertilization
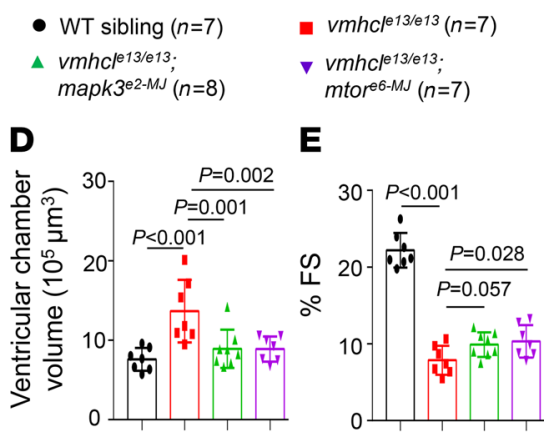

Figure 4. An FO screen of 11 target genes in 7 known CM pathways identified mapk3 and mtor as $\mathbf{2}$ therapeutic modifier genes for VEC. (A) Representative images of the heart area of FO fish at $3 \mathrm{dpf}$. The black star indicates severe edema, and red stars indicate mild edema. Scale bar: $300 \mu \mathrm{m}$. (B) Fluorescence immunostaining images using anti-myosin heavy chain 1 (F59) in the ventricles of (from top to bottom): WT controls, vmhcle13/e13, vmhcle13/213; mapk $3^{22-M 1}$, and

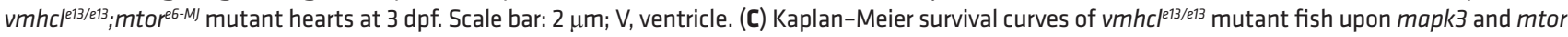
inhibition and WT controls. $n=15-17$; log-rank test. (D and E) VCV (D) and percent FS (E) of the vmhc/e13/e13 mutants after mapk3 and mtor inhibition compared to WT controls at $3 \mathrm{dpf} n=7-8$; data are presented as the mean \pm SD; 1 -way ANOVA. 
A
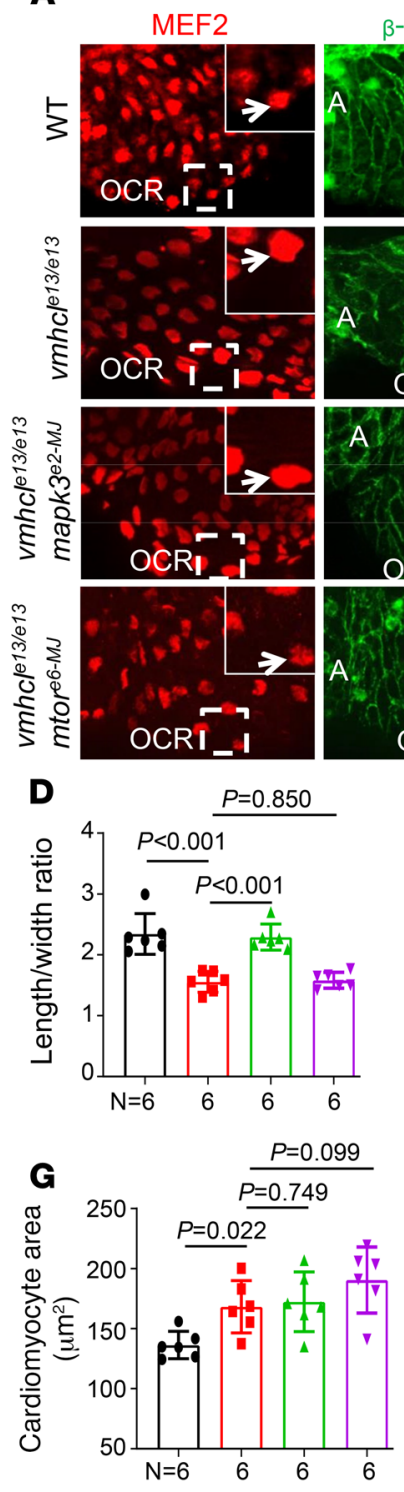

ß-Catenin
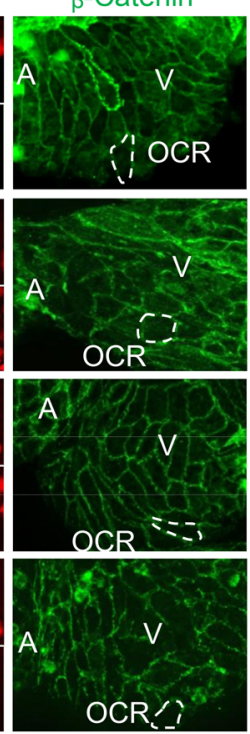

E
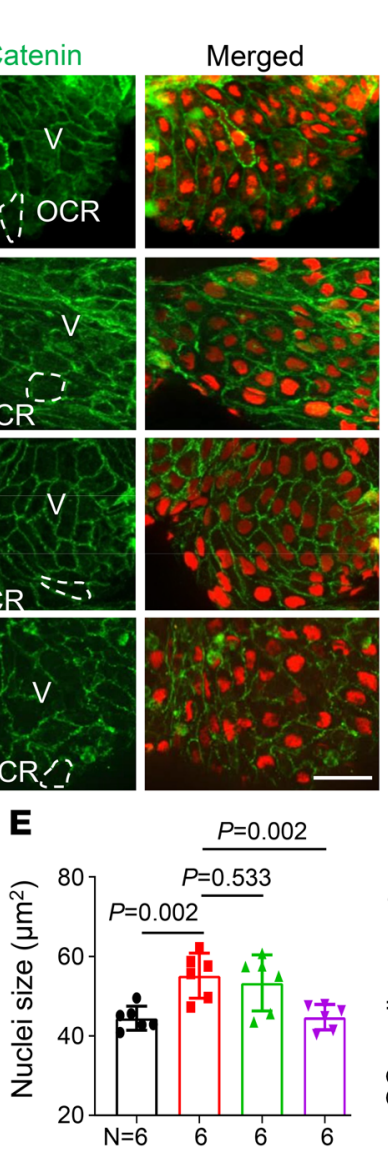

H

WT

cardiomyocyte $v m h c^{e 13 / e 13}$
B

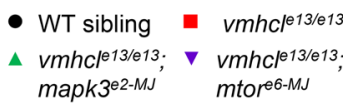
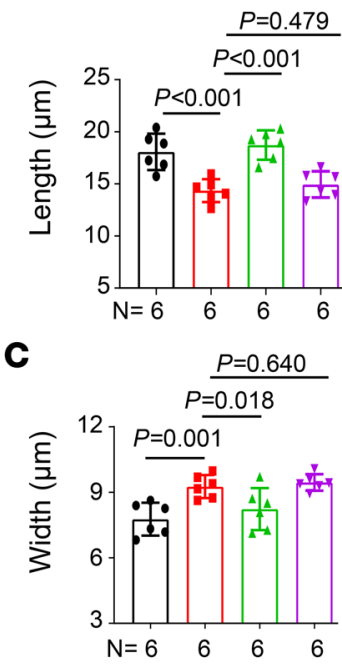

$\mathbf{F}$

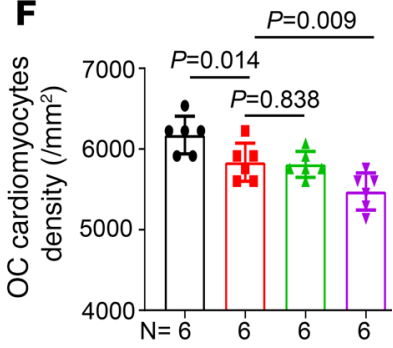

vmhc/e13/e13 mtore6-MJ

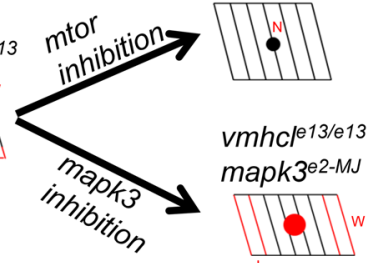

Figure 5. Distinct cellular effects of mtor and mapk3 inhibition on the VEC model. (A) Representative images of the nucleus and outline of cardiomyocytes were obtained by immunostaining with anti-myocyte enhancer factor-2 (red)

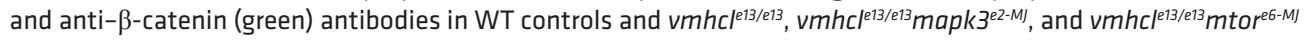
mutants at $3 \mathrm{dpf}$. The insets show the boxed areas at higher original magnification. The white arrows indicate nucle of the cardiomyocytes. Representative cardiomyocytes in the OCR are outlined by dashed white lines in panels with $\beta$-catenin staining. A, atrium; V, ventricle; scale bar: $25 \mu \mathrm{m}$. (B-C) Quantification of the cardiomyocyte length (B), cardiomyocyte width (C), cardiomyocyte length/width ratio (D), cardiomyocyte nuclei size (E), cardiomyocyte density $(\mathbf{F})$, and cardiomyocyte area (G) measured in these mutants and WT controls. $n=6$; data are presented as the mean \pm SD; 1-way ANOVA. (H) Schematics of different cellular changes observed upon mapk3 and mtor inhibition in the VEC model. Red indicates changes in cardiomyocyte length $(\mathrm{L})$, width $(\mathrm{W})$, or nuclei size $(\mathrm{N})$.

\section{Discussion}

$v m h c l$ depletion and haploinsufficiency lead to CM-like phenotypes in embryonic and adult zebrafish, respectively. In this study, through generating and characterizing the $v m h c l$ mutants, we presented zebrafish as a useful vertebrate model for deciphering MYH7 CM. The $v m h c^{13 /++}$ mutant fish developed several characteristics of $\mathrm{CM}$ at 8 months of age, including reduced cardiac pump function, increased density of the trabecular muscle, and reduced exercise capacity. Because sarcomeric defects have yet to be detected (data not shown), comprehensive cardiac phenotyping analyses of $v m h c^{1213 /+}$ heterozygous mutant fish at ages beyond 8 months are needed in the future. At the genome level, the $v m h c^{13 /+}$ mutant contains a small deletion in 
A

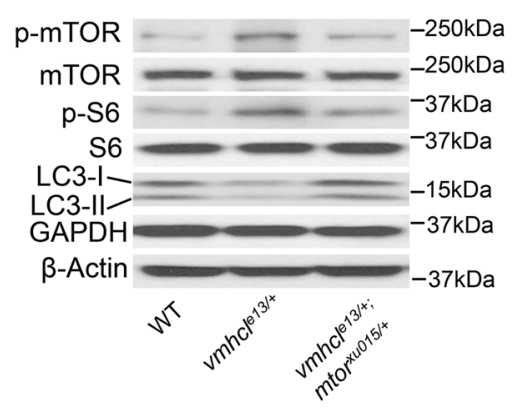

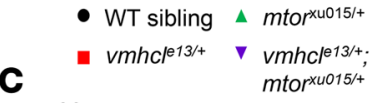

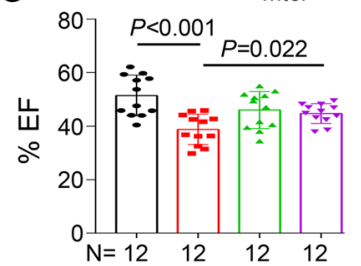

D

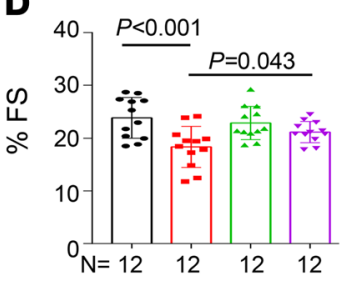

B
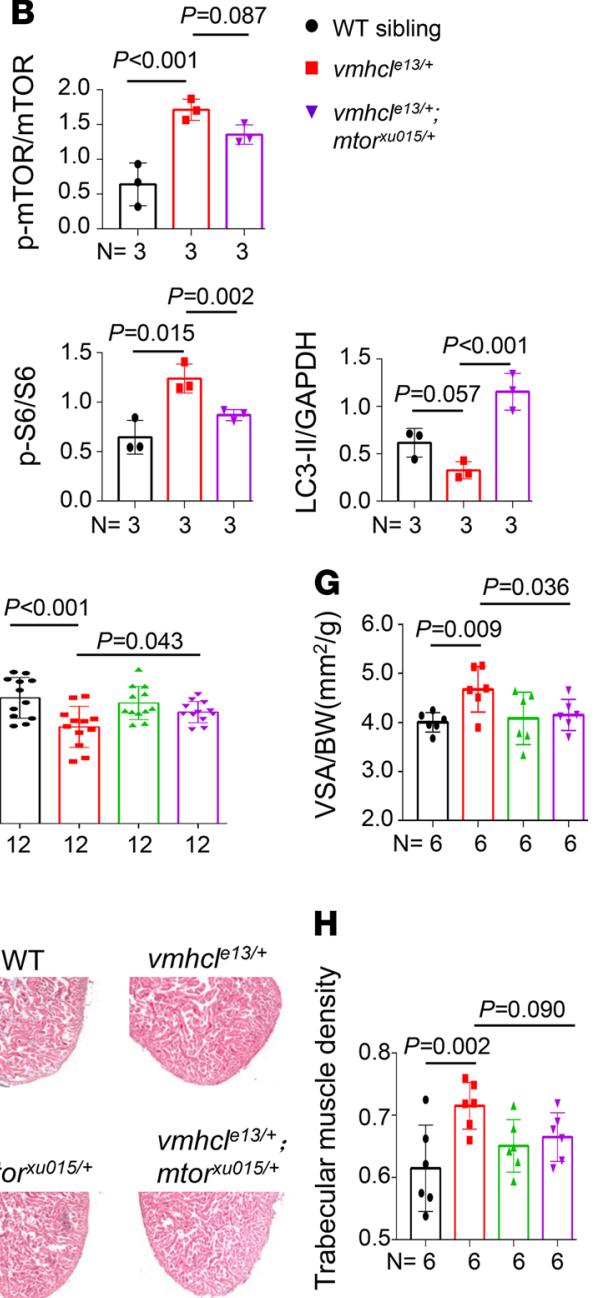

Figure 6. Therapeutic effects of mtor inhibition on the VAC model. (A and B) Representative Western blots showing the levels of proteins involved in mTOR signaling pathways in WT controls, vmhcle13/+, and vmhcle $^{13 /+} \mathrm{mtor}^{\mathrm{xu015/+}}$ mutants and quantification analysis. $n=3$ biological replicates; data are presented as the mean \pm SD; 1 -way ANOVA. (C and D) Quantification of the percent EF and FS using echocardiography in fish at 8 months. $n=12 ;$ data are presented as the mean \pm SD; 1-way ANOVA. (E and F) Representative images of isolated hearts and H\&E staining in the apex area in fish at 8 months. (G and $\mathbf{H}$ ) Quantification of the VSA normalized to BW and trabecular muscle density in these mutants and WT controls at 8 months. $n=6$; data are presented as the mean \pm SD; 1 -way ANOVA. Scale bars: $2 \mathrm{~mm}$ in $\mathbf{E}$ and $300 \mu \mathrm{m}$ in $\mathbf{F}$.

the exon 2 that shifts the reading frame and presumably results in early translational stop. At the mRNA level, we detected reduced levels of the $\mathrm{vmh} /$ transcript and compensatory expression of other myh homologs, particularly $v m h c$ and amhc. At the protein level, we noted reduced expression of overall Myh in the heart. Although we cannot eliminate the possibility of dominant negative effects incurred by a potentially truncated Vmhcl protein, our data strongly suggested that $v m h c l$ loss-of-function is sufficient to trigger CM-like responses in this animal.

An important discovery of the present work is to demonstrate that the efficient VEC model can be used for deciphering CM signaling pathways. An assessment of $7 \mathrm{CM}$ pathways via compound administration revealed 3 positive hits, 2 of which were subsequently validated by an independent genetic assessment of 11 target genes in all 7 signaling pathways. The therapeutic effects of both mTOR and MAPK inhibition were later confirmed in the VAC model. In fact, the possibility that zebrafish embryos can be used for deciphering the relationship between heart morphometry and cardiac function has been previously suggested by genetic studies of 2 other $m y h$ homologues, $v m h c$ and $a m h c(47,48)$. Unlike $v m h c l$, which is a predominant $m y h$ isoform in the adult ventricle, $v m h c$ is the predominant $m y h$ isoform in the embryonic ventricle, and $a m h c$ is the predominant $m y h$ isoform in the atrium $(40,47,48)$. Similar to $v m h c^{1213 / e 13}$, haf, a mutant 
affecting $v m h c$, displayed dramatically reduced ventricular contraction resulting in an enlarged ventricular chamber and enlarged cardiomyocytes (47). On the other hand, wea, a zebrafish mutant affecting amhc, has exhibited ablated atrial contractility resulting in a smaller ventricular chamber (49). The difference in the affected ventricular chamber size has been suggested to be a consequence of disrupted cardiac function in the ventricle and atrium, because the haf;wea double mutant rescued the increased ventricular size and the enlarged cardiomyocyte size (49). Together, our data strongly suggested that insights for MYH7 CM could be gleaned by studying myh mutants in zebrafish embryos.

mtor and mapk3 are 2 candidate therapeutic target genes for vmhcl-associated CM in zebrafish with different mechanisms. Based on both the assessment of $7 \mathrm{CM}$ pathway inhibitors and genetic testing of 11 candidate genes, we identified mtor inhibition as an approach to ameliorate VEC. We noted activated mTOR signaling in the VAC models and confirmed the therapeutic effects of mtor inhibition. Therapeutic effects similar to mtor inhibition have been observed on several other CM models, including anemia-induced CM (16), anthracycline-induced cardiotoxicity (16), bag3-associated DCM (14), and lamp2-associated HCM (13), suggesting that mtor signaling is a common pathological event in these CMs with distinct etiologies. Because aberrant protein quality control (PQC) is a common pathological event in many different CM types (50), it is possible that $m$ TOR inhibition exerts its therapeutic effects via modulating PQC within cardiomyocytes. Intriguingly, we noted enlarged cardiomyocyte nuclei in the VEC model, which was rescued by mTOR inhibition but not MAPK inhibition. We postulated that this change in nuclear size is a consequence of defective mTOR signaling and/or PQC.

In addition to mTOR signaling, both our compound-based and genetic tests also converge on a therapeutic function of MAPK signaling inhibition in the VEC model. Because ERK1/2 signaling was shown to be an important pathway that regulates the balance between eccentric and concentric growth of the heart (18), we propose that the ERK1/2 signaling pathway could be the key hypertrophic growth pathway that confers aberrant cardiac function to regulate the shape of cardiomyocytes.

Zebrafish are a valuable alternative animal model for MYH7 CM. Besides the well-established advantage of enabling larger-scale compound screening in zebrafish $(36-38,51)$, our study highlighted the feasibility of rapidly assessing modifier genes in F0 fish using the MMEJ-based CRISPR/Cas9 genome editing technology. Effective gene knockdown with predictable genetic lesions can be reliably achieved in F0. Results from the transient genetic analysis in F0 could then be validated in the F1 generation. Because the technology eliminates the need for genetic crosses of multiple generations that are typically required for genetic interaction studies, unprecedently high throughput can be achieved for assessing modifier genes and therapeutic strategies.

In summary, this study established zebrafish as a vertebrate model for studying $M Y H 7$ cardiomyopathy. Because of the conservation of the Myh6/Myh7 biology among zebrafish, larger mammals, and humans, but not rodents, our zebrafish VEC/VAC models possess great potential to serve as an alternative animal model to rodents. If inconsistent conclusions with rodents are noted, additional evidence from an $M Y H 7$ CM model in larger mammals, such as a rabbit model (22), is recommended. Therefore, we anticipate that the integration of zebrafish as an animal model for $M Y H 7 \mathrm{CM}$ will significantly accelerate mechanistic studies and therapeutic development.

\section{Methods}

Supplemental Methods are available online with this article. All supporting data and materials described within the article will be made available upon reasonable request.

Statistics. The unpaired 2-tailed Student's $t$ test was used to compare data between 2 groups. To assess differences among multiple groups, 1-way or 2-way ANOVA was used, as appropriate. The log-rank test was used to determine the difference in animal survival. All quantitative data are presented as the mean \pm $\mathrm{SD}$. The sample size ( $n$ ) represents the number of animals, unless otherwise specifically designated as the number of biological replicates. $P$ values of less than 0.05 were considered significant. All statistical analyses were performed using GraphPad Prism.

Study approval. Zebrafish (Danio rerio; WIK strain) were maintained on a 14-hour light/10-hour dark cycle at $28.5^{\circ} \mathrm{C}$. All animal study procedures were approved by Mayo Clinic Institutional Animal Care and Use Committee (protocol number: A3513) and performed in accordance with the Guide for the Care and Use of Laboratory Animals published by the US NIH (NIH publication no. 85-23, revised 1996). 


\section{Author contributions}

$\mathrm{HB}$, YD, and XX conceptualized the project. HB, YD, JL, YHS, MW, and PZ performed experiments and analyzed and interpreted data. YZ analyzed the statistics. HB, YD, XL, and XX wrote the manuscript. All authors reviewed the manuscript and discussed the work

\section{Acknowledgments}

This study was supported by grants from the National Institutes of Health (grants HL107304 and HL081753 to XX), the Mayo Foundation (to XX), and the China Scholarship Council (grant CSC201906370239 to HB).We also thank Beninio Gores and Kashia Stragey for managing the zebrafish facility.

Address correspondence to: Xiaolei Xu, Department of Biochemistry and Molecular Biology, Department of Cardiovascular Medicine, Mayo Clinic, 200 First Street SW, Rochester, Minnesota 55905, USA. Phone: 507.284.0685; Email: xu.xiaolei@mayo.edu.

YHS's present address is: Department of Molecular, Cell and Cancer Biology, University of Massachusetts Medical School, Worcester, Massachusetts, USA.

MW's present address is: Zhejiang Traditional Chinese Medicine Hospital, Hangzhou, China.

1. Maron BJ. Contemporary insights and strategies for risk stratification and prevention of sudden death in hypertrophic cardiomyopathy. Circulation. 2010;121(3):445-456.

2. Maron BJ, et al. Recommendations and considerations related to preparticipation screening for cardiovascular abnormalities in competitive athletes: 2007 update: a scientific statement from the American Heart Association Council on Nutrition, Physical Activity, and Metabolism: endorsed by the American College of Cardiology Foundation. Circulation. 2007;115(12):1643-1455

3. Ding Y, et al. A modifier screen identifies DNAJB6 as a cardiomyopathy susceptibility gene. JCI Insight. $2016 ; 1(14): 88797$.

4. Semsarian C, et al. New perspectives on the prevalence of hypertrophic cardiomyopathy. J Am Coll Cardiol. 2015;65(12):1249-1254.

5. Ahmad F, et al. The genetic basis for cardiac remodeling. Annu Rev Genomics Hum Genet. 2005;6:185-216.

6. Wang B, et al. Genetic analysis of monoallelic double MYH7 mutations responsible for familial hypertrophic cardiomyopathy. Mol Med Rep. 2019;20(6):5229-5238.

7. van Berlo JH, et al. Signaling effectors underlying pathologic growth and remodeling of the heart. J Clin Invest. 2013;123(1):37-45.

8. Pinilla-Vera $\mathrm{M}$, et al. Leveraging signaling pathways to treat heart failure with reduced ejection fraction. Circ Res. 2019;124(11):1618-1632.

9. Ashrafian H, et al. Disease pathways and novel therapeutic targets in hypertrophic cardiomyopathy. Circ Res. 2011;109(1):86-96.

10. Saxton RA, Sabatini DM. mTOR signaling in growth, metabolism, and disease. Cell. 2017;168(6):960-976.

11. Sciarretta S, et al. New insights into the role of mTOR signaling in the cardiovascular system. Circ Res. 2018;122(3):489-505.

12. Sciarretta S, et al. Mammalian target of rapamycin signaling in cardiac physiology and disease. Circ Res. 2014;114(3):549-564

13. Dvornikov AV, et al. Phenotyping an adult zebrafish lamp2 cardiomyopathy model identifies mTOR inhibition as a candidate therapy. J Mol Cell Cardiol. 2019;133:199-208.

14. Ding Y, et al. Haploinsufficiency of mechanistic target of rapamycin ameliorates bag3 cardiomyopathy in adult zebrafish. Dis Model Mech. 2019;12(10):dmm040154

15. Ramos FJ, et al. Rapamycin reverses elevated mTORC1 signaling in lamin A/C-deficient mice, rescues cardiac and skeletal muscle function, and extends survival. Sci Transl Med. 2012;4(144):144ra03.

16. Ding Y, et al. Haploinsufficiency of target of rapamycin attenuates cardiomyopathies in adult zebrafish. Circ Res. 2011;109(6):658-669.

17. Kehat I, Molkentin JD. Extracellular signal-regulated kinase 1/2 (ERK1/2) signaling in cardiac hypertrophy. Ann N Y Acad Sci. 2010;1188:96-102.

18. Kehat I, et al. Extracellular signal-regulated kinases 1 and 2 regulate the balance between eccentric and concentric cardiac growth. Circ Res. 2011;108(2):176-183.

19. Ulm S, et al. Targeted deletion of ERK2 in cardiomyocytes attenuates hypertrophic response but provokes pathological stress induced cardiac dysfunction. J Mol Cell Cardiol. 2014;72:104-116.

20. Ho CY, et al. Genotype and lifetime burden of disease in hypertrophic cardiomyopathy: insights from the sarcomeric human cardiomyopathy registry (SHaRe). Circulation. 2018;138(14):1387-1398.

21. Toepfer $\mathrm{CN}$, et al. Myosin sequestration regulates sarcomere function, cardiomyocyte energetics, and metabolism, informing the pathogenesis of hypertrophic cardiomyopathy. Circulation. 2020;141(10):828-842.

22. Lowey S, et al. Hypertrophic cardiomyopathy R403Q mutation in rabbit $\beta$-myosin reduces contractile function at the molecular and myofibrillar levels. Proc Natl Acad Sci U S A. 2018;115(44):11238-11243.

23. Lompre AM, et al. Expression of the cardiac ventricular alpha- and beta-myosin heavy chain genes is developmentally and hormonally regulated. J Biol Chem. 1984;259(10):6437-6446.

24. Geisterfer-Lowrance AA, et al. A molecular basis for familial hypertrophic cardiomyopathy: a beta cardiac myosin heavy chain gene missense mutation. Cell. 1990;62(5):999-1006.

25. Tyska MJ, et al. Single-molecule mechanics of R403Q cardiac myosin isolated from the mouse model of familial hypertrophic cardiomyopathy. Circ Res. 2000;86(7):737-744. 
26. Cuda G, et al. The in vitro motility activity of beta-cardiac myosin depends on the nature of the beta-myosin heavy chain gene mutation in hypertrophic cardiomyopathy. J Muscle Res Cell Motil. 1997;18(3):275-283.

27. Marian AJ, et al. A transgenic rabbit model for human hypertrophic cardiomyopathy. J Clin Invest. 1999;104(12):1683-1692.

28. Nagueh SF, et al. Evolution of expression of cardiac phenotypes over a 4-year period in the beta-myosin heavy chain-Q403 transgenic rabbit model of human hypertrophic cardiomyopathy. J Mol Cell Cardiol. 2004;36(5):663-673.

29. Moore JR, et al. Understanding cardiomyopathy phenotypes based on the functional impact of mutations in the myosin motor. Circ Res. 2012;111(3):375-385.

30. Xu X, et al. Cardiomyopathy in zebrafish due to mutation in an alternatively spliced exon of titin. Nat Genet. 2002;30(2):205-209.

31. Sehnert AJ, et al. Cardiac troponin T is essential in sarcomere assembly and cardiac contractility. Nat Genet. 2002;31(1):106-110.

32. Dvornikov AV, et al. Phenotyping cardiomyopathy in adult zebrafish. Prog Biophys Mol Biol. 2018;138:116-125.

33. Shih YH, et al. Exon- and contraction-dependent functions of titin in sarcomere assembly. Development. 2016;143(24):4713-4722.

34. Huttner IG, et al. A-band titin truncation in zebrafish causes dilated cardiomyopathy and hemodynamic stress intolerance. Circ Genom Precis Med. 2018;11(8):e002135.

35. Ata $\mathrm{H}$, et al. Robust activation of microhomology-mediated end joining for precision gene editing applications. PLoS Genet. 2018;14(9):e1007652.

36. Liu Y, et al. Visnagin protects against doxorubicin-induced cardiomyopathy through modulation of mitochondrial malate dehydrogenase. Sci Transl Med. 2014;6(266):266ra170.

37. Becker JR, et al. In vivo natriuretic peptide reporter assay identifies chemical modifiers of hypertrophic cardiomyopathy signalling. Cardiovasc Res. 2012;93(3):463-470.

38. Yang J, et al. Cessation of contraction induces cardiomyocyte remodeling during zebrafish cardiogenesis. Am J Physiol Heart Circ Physiol. 2014;306(3):H382-H395.

39. Ding Y, et al. Trapping cardiac recessive mutants via expression-based insertional mutagenesis screening. Circ Res. 2013;112(4):606-617.

40. Shih YH, et al. Cardiac transcriptome and dilated cardiomyopathy genes in zebrafish. Circ Cardiovasc Genet. 2015;8(2):261-269

41. Wilkins BJ, Molkentin JD. Calcium-calcineurin signaling in the regulation of cardiac hypertrophy. Biochem Biophys Res Commun. 2004;322(4):1178-1191.

42. Robinson BL, et al. Cyclosporine exacerbates ketamine toxicity in zebrafish: mechanistic studies on drug-drug interaction. $J$ Appl Toxicol. 2017;37(12):1438-1447.

43. Kho C, et al. Small-molecule activation of SERCA2a SUMOylation for the treatment of heart failure. Nat Commun. 2015;6:7229.

44. Campbell LJ, Jensen AM. Phosphodiesterase inhibitors sildenafil and vardenafil reduce zebrafish rod photoreceptor outer segment shedding. Invest Ophthalmol Vis Sci. 2017;58(13):5604-5615.

45. Hoshijima K, et al. Highly efficient CRISPR-Cas9-based methods for generating deletion mutations and F0 embryos that lack gene function in zebrafish. Dev Cell. 2019;51(5):645-657.

46. Wu RS, et al. A rapid method for directed gene knockout for screening in G0 zebrafish. Dev Cell. 2018;46(1):112-125.

47. Auman HJ, et al. Functional modulation of cardiac form through regionally confined cell shape changes. PLoS Biol. 2007;5(3):e53.

48. Pradhan A, et al. FGF signaling enforces cardiac chamber identity in the developing ventricle. Development. 2017;144(7):1328-1338.

49. Berdougo E, et al. Mutation of weak atrium/atrial myosin heavy chain disrupts atrial function and influences ventricular morphogenesis in zebrafish. Development. 2003;130(24):6121-6129.

50. Islam M, et al. Come together: protein assemblies, aggregates and the sarcostat at the heart of cardiac myocyte homeostasis Front Physiol. 2020;11:586.

51. Baxendale S, et al. Identification of compounds with anti-convulsant properties in a zebrafish model of epileptic seizures. Dis Model Mech. 2012;5(6):773-784. 